\title{
A rare case of jejunal diverticulitis
}

\author{
S Tilakaratne ${ }^{1}$, W Coomaraswamy ${ }^{2}$, SD Ratnapala ${ }^{3}$. \\ ${ }^{1}$ Consultant Surgeon, ${ }^{2}$ Senior Registrar in Surgery, ${ }^{3}$ Registrar in Surgery, Teaching Hospital, Karapitiya, Galle. \\ e-mail address of the corresponding author,Dr.Devaka Rathnapala: sahandoc@hotmail.com
}

\section{Introduction}

Jejunal diverticulitis is a rare condition which usually occurs in the elderly. Autopsy studies have revealed an incidence between $1.3 \%$ and $4.6 \%$ and radiological studies indicated an incidence between $0.02 \%$ and $2.3 \%{ }^{1}$.

The condition is difficult to diagnose because its symptoms can mimic other conditions of acute abdomen. Perforation and other complications of diverticula are common in this condition ${ }^{2}$. We report a patient with jejunal diverticulitis who was successfully treated surgically.

\section{Case report}

A 77 year-old woman presented with a three day history of generalised abdominal pain, a two day history of constipation and loss of appetite and fever. She had vomited twice. She is on regular treatment for hypertensive. On physical examination, she was ill looking with a temperature of $102 \mathrm{~F}$, pulse rate of 96 beats/min and blood pressure of 110/80 mmHg. Abdomen was mildly distended with generalised tenderness. No masses were felt. Bowel sounds were sluggish. Per rectal examination and examination of other systems were unremarkable.

Investigations showed a white cell count of $28,000 / \mathrm{mm}^{3}$ with neutrophil leucocytosis. Serum electrolytes were normal. X-ray abdomen showed faecal loading of the colon. There were no distended bowel loops. There was no air under the diaphragm on erect chest X-ray.

Possible diagnosis at this moment was generalised peritonitis due to perforated appendix, perforated peptic ulcer, perforated colonic tumour or colonic diverticulitis. After initial stabilisation of the patient an exploratory laparotomy was carried out.

A midline exploratory laparotomy was performed and there was free exudative fluid in the peritoneal cavity with fibrin coating of the small bowel. The jejunum had multiple diverticula arising from the mesenteric border and 3 diverticula adjacent to each other were highly inflammed. The jejunum segment with diverticula was around $40 \mathrm{~cm}$ in length. Resection of the involved segment was carried out and an end to end anastomosis was done using Vicryl. Rest of the small bowel and colon was normal. Peritoneal lavarge was done and a drain was placed. Mass closure of abdomen was done using Nylon.

Patient recovered well and was discharged home tolerating a normal diet on $6^{\text {th }}$ postoperative day.

\section{Discussion}

Jejunal diverticulosis was first reported by Sir Astley Paston Cooper in $1807^{3}$. Both males and females are equally affected. These are usually false diverticula and occur along the mesenteric border of the intestine. Prompt recognition is important in symptomatic elderly patients due to risk of perforation. Jejunal diverticula are usually asymptomatic but can present as acute abdomen. Complications and mode of presentation include acute inflammation, perforation with peritonitis, mechanical obstruction, fistula formation and abscess formation ${ }^{4}$.

Jejunal diverticulosis is often difficult to diagnose. Standard upper gastrointestinal contrast studies are helpful which may show contrast filled outpouchings characteristic of diverticulosis. CT scan of the abdomen with oral contrast is of great value since it will demonstrate diverticula and in acute inflammation may show mesenteric stranding, abscess or free air around the divertucula site ${ }^{3}$. Plain erect abdominal X-rays may show air-fluid levels in the diverticula. An elevated WBC count will indicate an inflammatory process. 
Management of jejunal diverticula depends on a patient's symptoms and presentation. Asymptomatic diverticula found incidentally during laparotomy should be left alone. Surgical resection is the standard care for symptomatic patients with inflammed, complicated diverticular disease. Surgery involves resection of the affected bowel segment and primary end to end anastomosis. Laparoscopic exploration and resection is also practiced in certain centres. Extensive resection of jejunum will lead to short bowel syndrome and therefore only the affected part should be resected.

\section{Conclusion}

Jejunal diverticular disease is a rare condition usually occurring in the elderly which is difficult to diagnose. It is of great importance that the whole bowel is carefully examined during laparotomy in an elderly patient who presents with an acute abdomen since this condition can be easily missed. Surgical resection of the involved segment with primary end to end anastomosis is the method of choice to treat this condition.

\section{References}

1. Ross CB, Richards WO et al. Diverticular disease of the jejunum and its complications. Am Surg 1990; 56(5);319324.

2. R.C.G. Russell, Norman S. Williams, Christopher JKB. The small and large intestine. In: Bailey and Love's Short Practice of Surgery: $24^{\text {th }}$ edition, Arnold, London, 2005: pg 1158-9.

3. Goshtasby P, Tiruchelvum V. Perforated jejunal diverticulitis, Surg Rounds, Aug 2006.

4. Robbins, Cotran, Kumar. Gatrointestinal tract. Pathologic Basis of Disease: $5^{\text {th }}$ edition. W.B. Sounders. p331-2. 